\title{
Using Time-Series and Spatial Analysis (Math-Physical Medicine) to Investigate the Relationships among Weight, Blood Pressure, Glucose, Food and Exercise
}

Gerald C Hsu

EclaireMD Foundation, USA

\section{Introduction}

The author uses his own type 2 diabetes metabolic conditions control as a case study using the "math-physical medicine" approach of a non-traditional methodology in medical research.

Math-physical medicine (MPM) starts with the observation of the human body's physical phenomena (not biological or chemical characteristics), collecting elements of the disease related data (preferring big data), utilizing applicable engineering modeling techniques, developing appropriate mathematical equations (not just statistical analysis), and finally predicting the direction of the development and control mechanism of the disease.

The author uses the MPM approach to investigate the relationships between five pairs:

1. Weight vs. blood pressure (BP)

2. Weight vs. glucose - both fasting plasma glucose (FPG) and postprandial plasma glucose (PPG)

3. BP and glucose

4. $P P G$ and carbs/sugar

5. PPG and exercise

\section{Method}

The selected time periods involve two separated sets: $2 / 1 / 2014$ - 11/2/2018 with 17,350 metabolic syndrome (weight, BP, and glucose) data and 10/1/2015 - 11/2/2018 with 10,252 food and exercise data. This paper utilized two statistical tools, i.e. timeseries (similar to EKG chart) and spatial analysis (in a twodimensional $\mathrm{x}$ and $\mathrm{y}$ space, without "time" factor). In the time series graphs, if the correlation coefficient $(\mathrm{R})$ is greater than $60 \%$, then these two curves are highly correlated. Correlation can only be calculated for two sets of data (curves). In spatial analysis, if the "data cloud" is skewed (i.e. slope is much

\section{Correspondence author}

\section{Gerald C Hsu}

EclaireMD Foundation

USA

Submitted : 07 April 2020 ; Published : 17 April 2020

greater than zero), then these two sets of data are correlated $[1-5]$.

\section{Results}

The correlation results are as follows:

1. Weight vs. BP: $68 \%$

2. Weight vs. glucose: $78 \%$

3. Glucose vs. BP: $85 \%$

4. PPG vs. carbs/sugar: $+55 \%$

5. PPG vs. post-meal walking: $-66 \%$

6. Weight is correlated with FPG, not with PPG.

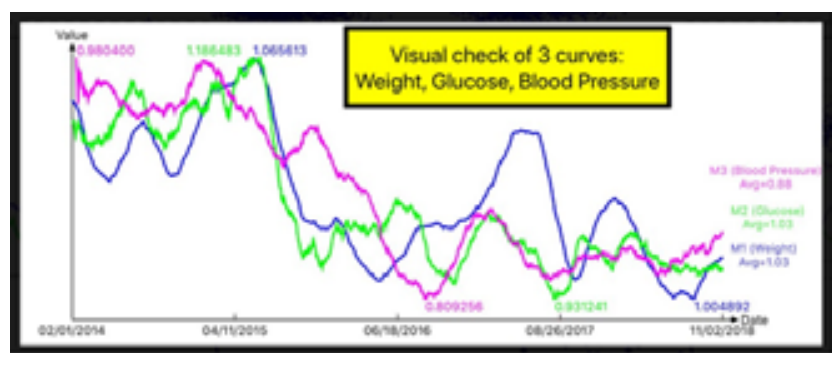

Figure 1: Visual check of three curves - Weight, Glucose, and Blood Pressure 


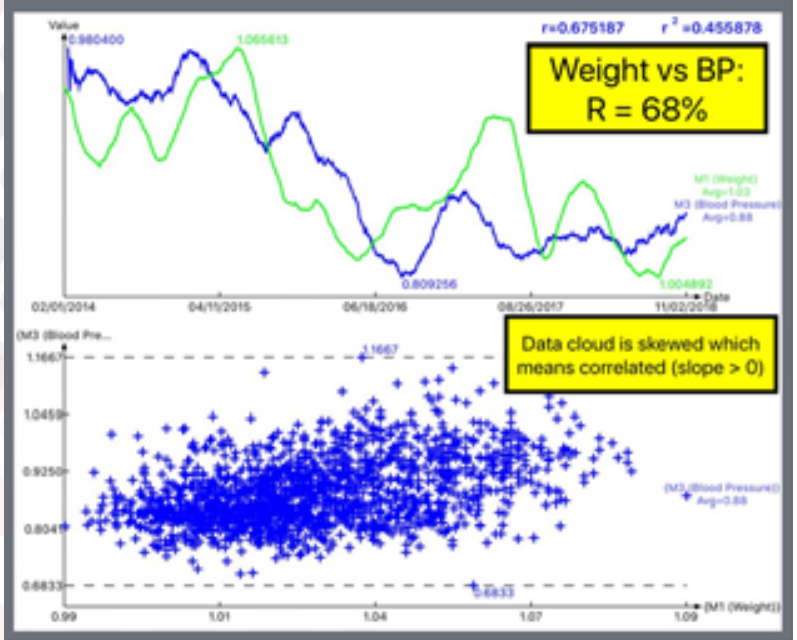

Figure 2: Weight vs. BP, $\mathrm{R}=68 \%$

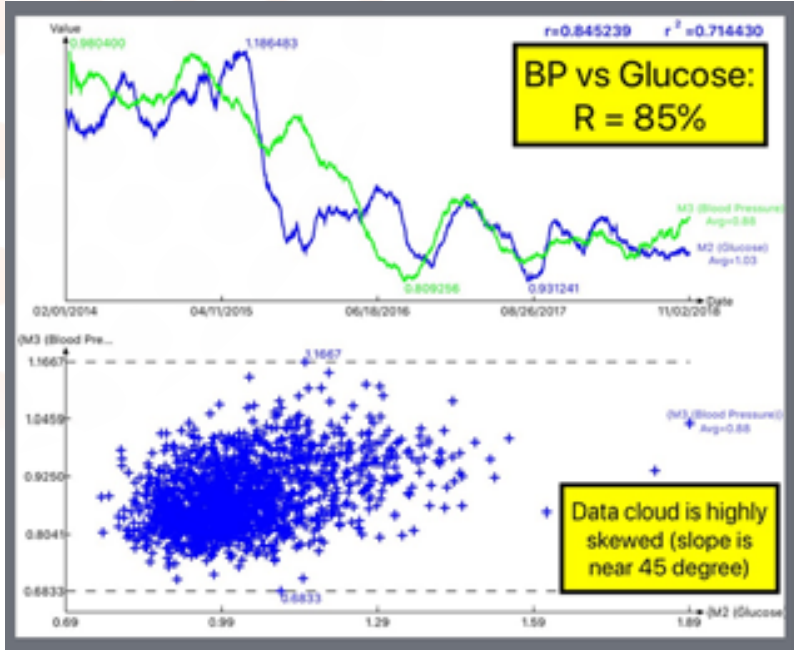

Figure 3: BP v. Glucose, $\mathrm{R}=85 \%$

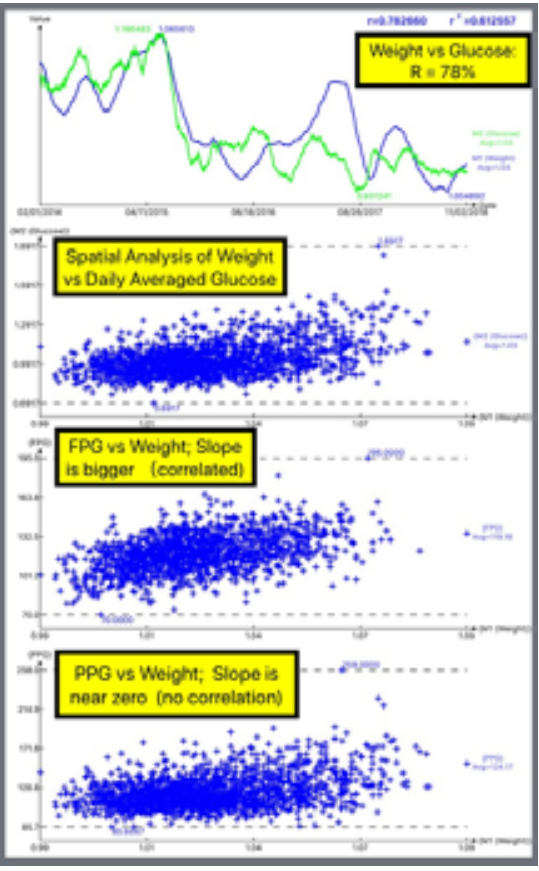

Figure 4: Spatial Analysis, FPG vs. Weight, and PPG vs. Weight

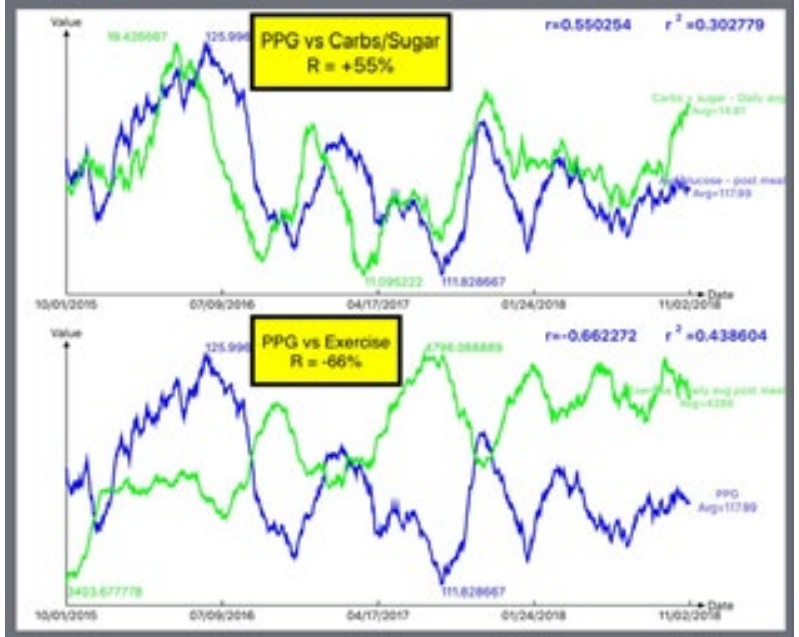

Figure 5: PPG vs. Carbs/Sugar, PPG vs. Exercise

\section{Conclusion}

All correlations among weight, BP, glucose, carbs/sugar, exercise are strong; therefore, these three metabolic syndromes are related to each other. PPG is highly influenced by both food and exercise. The author did not discover anything new from this study. However, he just used math-physical medicine and big data analytics to provide some additional mathematical and quantitative proof to the healthcare community paradigm.

\section{References}

1. Hsu Gerald C (2018) Using Math-Physical Medicine to Control T2D via Metabolism Monitoring and Glucose Predictions. Journal of Endocrinology and Diabetes 1: 1-6.

2. Hsu Gerald C (2018) Using Math-Physical Medicine to Analyze Metabolism and Improve Health Conditions. Video presented at the meeting of the 3rd International Conference on Endocrinology and Metabolic Syndrome 2018, Amsterdam, Netherlands.

3. Hsu Gerald C (2018) Using Signal Processing Techniques to Predict PPG for T2D. International Journal of Diabetes \& Metabolic Disorders 3: 1-3.

4. Hsu Gerald C (2018) Using Math-Physical Medicine and Artificial Intelligence Technology to Manage Lifestyle and Control Metabolic Conditions of T2D. International Journal of Diabetes \& Its Complications 2: 1-7.

5. Hsu Gerald C (2018) A Clinic Case of Using MathPhysical Medicine to Study the Probability of Having a Heart Attack or Stroke Based on Combination of Metabolic Conditions, Lifestyle, and Metabolism Index. Journal of Clinical Review \& Case Reports 3: 1-2.

Copyright: $(02020$ Gerald C Hsu. This is an open-access article distributed under the terms of the Creative Commons Attribution License, which permits unrestricted use, distribution, and reproduction in any medium, provided the original author and source are credited. 